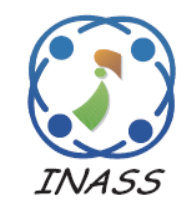

\title{
Adaptive Neuro-Fuzzy Interference System Modelling of EDM Process Using CNT Infused Copper Electrode
}

\author{
Prabhu Sethuramalingam $^{1 *} \quad$ Oliver Nesa Raj Sundararaj ${ }^{1}$ \\ ${ }^{1}$ Department of Mechanical Engineering, SRM University, Chennai, India \\ * Corresponding author's Email: prabhume@yahoo.co.in
}

\begin{abstract}
This study deals with the experimental investigation of the machining characteristics of AISI D2 Tool Steel on the EDM process by using Carbon Nanotube (CNT) infused nano copper electrode, and checking the improvement in machinability characteristics like Material Removal Rate (MRR), Electrode wear rate (EWR) and Surface finish (SR). The work material is spark machined with pure copper and CNT-infused copper electrodes by varying parameters on an EDM. Experiment work is conducted based on Taguchi L9 orthogonal array. The various results of the machining characteristics of with and without CNT are compared and analyzed by means of Adaptive Neuro-Fuzzy Inference System (ANFIS) approach; Analysis of variance (ANOVA) and F-test are used to check the validity of regression model and determine the significant parameter affecting the surface roughness. The output values are found to be close to predicted values with an error of less than 5\%.
\end{abstract}

Keywords: Multiwall carbon nanotube, Taguchi analysis, CNT copper electrode, ANOVA, Fuzzy logic, ANFIS

\section{Introduction}

Electrical discharge machining (EDM), once considered a non-traditional machining process, has been replacing conventional machining operations and is now a well-established the preferred machining option in many manufacturing industries throughout the world. Modern EDMs are capable of machining geometrically complex and/or hard material components that need to be precise and are difficult-to-machine, such as heat treated tool steels, composites, super alloys, ceramics, etc. With such increasing use of EDM in high-precision processes, the demand for further improvement, that is better machining characteristics, better surface finish and reduced wear characteristics arises.

\subsection{Previous work}

Kiyak and Aakır [1] conducted an experimental study on AISI P20 tool steel with Electrical Discharge Machining (EDM). By varying the process parameters, the optimum combination of parameters to obtain the highest surface finish quality was determined. Cheharon et al., [2] examined the performance of copper and graphite electrodes on EDM with XW42 tool steel and proved that copper offers a higher MRR with reduced wear whilst graphite offers better finish. Guu [3] investigated the surface morphology, surface roughness and micro-crack of AISI D2 tool steel machined by the Electrical discharge machining process and conducted an analysis by means of the AFM technique. Guu et al., [4] studied the surface characteristics and machining damages in AISI D2 tool steel caused by EDM in terms of machining parameters. Based on the experimental data, an empirical model of tool steel was also proposed. Prabhu and Vinayagam [5] noted that recent developments in EDM have opened up new avenues for ensuring nano surface finish for hard and brittle materials with high tolerance and accuracy. A single-wall carbon nanotube (SWCNT) has high electrical conductivity. The carbon nanotube was mixed with dielectric in EDM process to analyze the surface characteristics like surface roughness and micro cracks in AISI D2 tool steel workpiece materials which are commonly used in 
moulds and dies. Experimental results indicated that an excellent machined finish could be obtained by setting the machine parameters at low pulse energy. Furthermore, the SEM application that yielded information about the depth of the micro cracks was particularly important in the post treatment of AISI D2 tool steel machined by EDM. Later, the parameters were optimized using MINITAB 15 software and regression equations were compared, with and without SWCNTs.

Kuang-Chyi Lee et al. [6] proposed a method using ANFIS to establish the relationship between actual surface roughness and texture features of the surface image. The input parameters of the training model were spatial frequency, arithmetic mean value and standard deviation of gray levels from the surface image, without involving cutting parameters. Experiments demonstrated the validity and effectiveness of fuzzy neural networks for modeling and estimating surface roughness.

Kuntal Maji and Dilip Kumar Pratihar [7] developed the input output relationships of an electrical discharge machining process established both in forward as well as reverse directions using adaptive network-based fuzzy inference system. Three input parameters, such as peak current, pulseon-time and pulse-duty-factor, and two outputs, namely material removal rate and surface roughness were considered for the said mappings. A batch mode of training was adopted with the help of 1000 data for the developed adaptive network-based fuzzy inference system, which was designed using linear and non-linear membership function distributions of the input variables.

Tsai and Wang [8] tried to predict surface finish in Electrical Discharge Machining (EDM) process using various neural networks and ANFIS, and compared their performances. . Yilmaz, et al., [9] used a user friendly fuzzy logic-based system for the selection of EDM process parameters. An ANFIS model was developed by Caydas et al., [10] for the prediction of white layer thickness and average surface roughness as the function of the process parameters. Pulse duration, open-circuit voltage, dielectric flushing pressure and wire feed rate were taken as the model's inputs. The model's predictions were compared with the experimental results for verification and were found to be satisfactory. Tsai and Wang [11] used various neural network models and a neuro-fuzzy model named ANFIS for predicting metal removal rate (MRR) in EDM process. The developed models were compared in terms of their performances. The ANFIS model with bell-shaped membership function distributions for the input variables was found to yield the best results amongst all. Minggang Dong and Ning Wang [12] proposed to model surface roughness with ANFIS and leave-one-out cross-validation (LOO-CV) approach. This approach focuses on both architecture and parameter optimization in order to improve the prediction accuracy in end milling process.

Deepak Joshi et al., [13] applied a first-order Sugeno type ANFIS to predict the knee angle from contra lateral knee angle with different walking speeds. The other two variables to train ANFIS were derivative of knee angle trajectory and the best fit curve equation between the trajectories of both knee angles. Joshi et al., [14] reported an intelligent approach for process modeling and optimization of EDM. Physics based process modeling using finite element method (FEM) was integrated with soft computing techniques like artificial neural networks (ANN) and genetic algorithm (GA) to improve prediction accuracy of the model with less dependency on the experimental data. Yih-fong Tzeng et al., [15] described the application of fuzzy logic analysis coupled with Taguchi methods to optimize the precision and accuracy of the highspeed EDM process. A fuzzy logic system is used to investigate relationships between the machining precision and accuracy for determining the efficiency of each parameter design of the Taguchi dynamic experiments. From the fuzzy inference process, the optimal process conditions for the highspeed EDM process can be easily determined. Hashmi et al., [16] developed Fuzzy-logic principles and applied them for selecting cutting conditions in machining operations. The materials data used for theoretical calculations were for medium-carbon leaded steel and free-machining carbon wrought steel. A fuzzy model was developed for carrying out these calculations. In their model, the fuzzy-metric arcs overlapped at $50 \%$.

Grzegorz Skrabalak et al., [17] presented a simplified model for estimating current of electrochemical dissolution and electrochemical discharge machining process (ECDM). Based on their model they also presented an attempt to adapt fuzzy-logic controller for the ECDM process.

Rajasekaran et al., [18] examined the influence of machining parameters combination so as to obtain a good surface finish in turning of carbon fiber reinforced plastics (CFRP) composite by cubic boron nitride cutting tool and to predict the surface roughness values using fuzzy modeling. The results indicated that the fuzzy logic modeling technique could be effectively used for the prediction of surface roughness in machining of CFRP composites. Hashmi et al., [19] described an 
adjustment approach applied to a fuzzy logic based model for selecting cutting speed when the machining data for speed ranges between the different depths cut did not overlap. For this study data for two different materials, four different types of cutting tools were used to illustrate the adjustment approach. In material type one, the cutting speed ranges for different depths of cut overlapped and when a fuzzy logic based model was applied, it produced the cutting speed data providing good correlation with handbook data.

In this paper, carbon nanotube mixed copper electrode tool is used in the EDM process to analyze the surface characteristics of AISI D2 tool steel material. Until now, not much work has been carried out using CNT based electrode tool in EDM machining. Carbon nanotubes are known to improve the strength, wear resistance and durability of metals and are hence incorporated in this study with the expectance of it being able to reduce the wear of electrodes and to enhance their life without compromising on either the dimensional or shape accuracy of the machined part. An improved approach is proposed to model surface roughness with ANFIS by using CNT based electrode tool using Taguchi design of experimental techniques. Scanning electron microscope (SEM) is used to analyze the surface morphology of AISI D2 tool steel.

Table 1. Properties and specifications of AISI D2 Tool

$$
\text { steel }
$$

\begin{tabular}{|c|c|}
\hline Category & Steel \\
\hline Class & Tool steel \\
\hline Type & $\begin{array}{l}\text { High-carbon, high- } \\
\text { chromium cold work steel }\end{array}$ \\
\hline Density $\left(\mathrm{kg} / \mathrm{m}^{3}\right)$ & 7.74 \\
\hline Poisson's Ratio & $0.27-0.30$ \\
\hline Elastic Modulus (GPa) & $190-210$ \\
\hline Thermal Expansion $\left({ }^{\circ} \mathrm{C}\right)$ & $10.4 \times 10^{-6}$ \\
\hline Hardness (HRC) & $55-62$ \\
\hline
\end{tabular}

Table 2. Composition of AISI D2 Tool steel

\begin{tabular}{|c|c|c|c|c|c|}
\hline \multirow{2}{*}{ Element } & $\mathrm{C}$ & $\mathrm{Si}$ & $\mathrm{Mn}$ & $\mathrm{P}$ & $\mathrm{S}$ \\
\cline { 1 - 4 } $\mathrm{Wt}(\%)$ & 1.66 & 0.436 & 0.201 & 0.03 & 0.029 \\
\cline { 1 - 4 } Element & $\mathrm{Cr}$ & $\mathrm{Mo}$ & $\mathrm{V}$ & $\mathrm{Fe}$ & \multirow{2}{*}{} \\
\cline { 1 - 4 } $\mathrm{Wt}(\%)$ & 11.59 & 0.232 & 0.042 & Balance & \\
\hline
\end{tabular}

\subsection{AISI D2 tool steel}

AISI D2 is an air hardening, high-carbon, highchromium tool steel. It has high wear and abrasion resistant properties. AISI D2 tool steel shows little distortion on after correct hardening. D2 steel having high chromium content gives it mild corrosion resisting properties in the hardened condition. The physical properties and chemical composition of AISI D2 tool steel were reported by Microlab, Ambattur, India as shown in Table 1 and 2. AISI D2 tool steel is used mainly in stamping or forming dies, punches, forming rolls, knives, slitters, shear blades, gauges, mandrels, tools, scrap choppers, tyre shredders etc.

\subsection{CNT-infused copper electrodes}

Owing to the special characteristics of multi walled CNT such as improving electrical conductivity, increasing strength and increasing wear resistance, CNT was prepared by mixing copper in the powder form, and infusing it through the stir casting process. The copper-CNT electrode that was cast out and machined to shape is depicted below in Fig.1. In spark-erosion machining on the EDM, copper is the most predominantly used electrode material as it has been proven by many researchers to be the best and most suitable electrode based on overall performance. It offers a good surface finish without compromising on the MRR whilst offering a reduced EWR. Though it is usually employed in roughing operations it can also produce a good surface finish. The chemical composition of CNT infused electrode is shown in Tables 3 and 4.

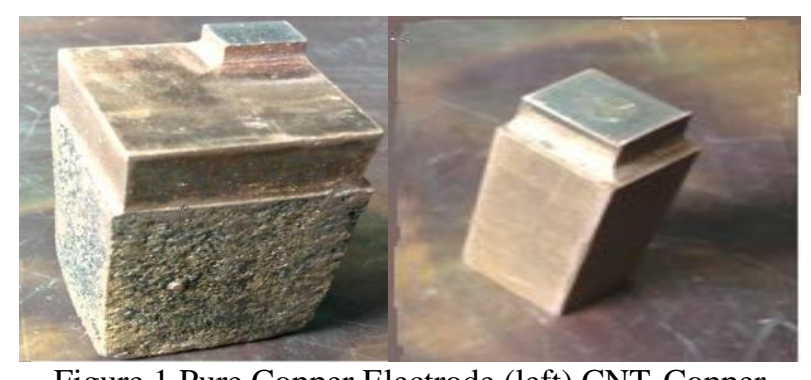

Figure.1 Pure Copper Electrode (left) CNT-Copper Electrode (right)

Table 3. Composition of Copper CNT Electrode

\begin{tabular}{|c|c|c|c|c|c|}
\hline Element & $\mathrm{C}$ & $\mathrm{Pb}$ & $\mathrm{Fe}$ & $\mathrm{Sn}$ & $\mathrm{Cu}$ \\
\hline $\mathrm{Wt}(\%)$ & 0.701 & 0.058 & 0.782 & 0.033 & 98.38 \\
\hline
\end{tabular}


Table 4. Composition of pure Copper Electrode

\begin{tabular}{|l|c|c|c|c|}
\hline Element & $\mathrm{Sn}$ & $\mathrm{Fe}$ & $\mathrm{Bi}$ & $\mathrm{Cu}$ \\
\hline $\mathrm{Wt}(\%)$ & 0.055 & 0.007 & 0.002 & 99.91 \\
\hline
\end{tabular}

\section{Fuzzy modelling of surface roughness based on ANFIS}

\subsection{Adaptive neuro fuzzy interference system architecture}

Adaptive Neuro Fuzzy Interference System (ANFIS) are a class of adaptive networks that are functionally equivalent to fuzzy inference systems and it represents Sugeno type fuzzy models used as a hybrid learning algorithm. The ANFIS architecture has 5 layers in which rectangular box have variable values and circle box has fixed values, which are shown in Fig.2.

Layer1: Ol, i is the output of the $i$-th node of layer l. Every node $i$ in this layer is an adaptive node with a node function. $\mathrm{O} 1, \mathrm{i}=\mu \mathrm{Ai}(\mathrm{x})$ for $i=1,2$, or $\mathrm{O}_{1, \mathrm{i}}=\mu \mathrm{Bi}-2(\mathrm{x})$ for $i=3,4, \mathrm{x}, \mathrm{y}$ (or $\mathrm{z}$ ) is the input node $\mathrm{i}$ and $\mathrm{Xi}, \mathrm{Yi}$ and $\mathrm{Zi}$ is are a linguistic label associated with this node, Therefore O1,i is the membership grade of a fuzzy set (X1,X2,X3, $\mathrm{Y} 1, \mathrm{Y} 2, \mathrm{Y} 3, \mathrm{Z1}, \mathrm{Z} 2, \mathrm{Z} 3)$. Here input parameters for EDM machining are: $\mathrm{X}$-pulse on-time, $\mathrm{Y}$-pulse off-time and $\mathrm{Z}$ - pulse current.

Layer 2: Every node in this layer is a fixed node labeled PRO. The output is the product of all the incoming signals.

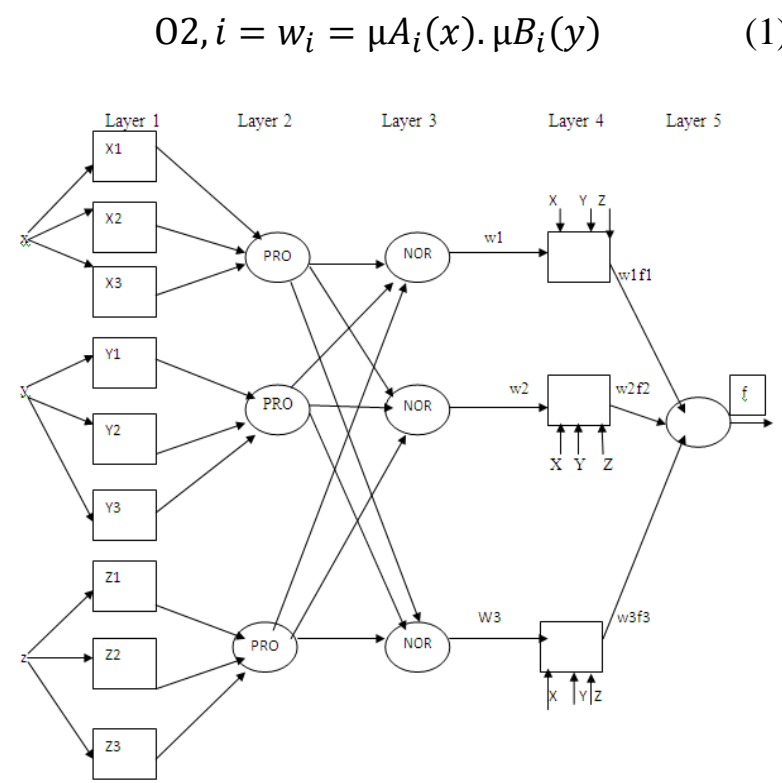

Figure.2 ANFIS architecture for forward mapping of WEDM process $i=1,2$, each node represents the fire strength of the rule. Any other T-norm operator that performs the AND operator can be used.

Layer 3: Every node in this layer is a fixed node labeled NOR. The $i$-th node calculates the ratio of the ith rule's firing strength to the sum of all rule's firing strengths.

$$
03, i=w_{i}=\frac{w_{i}}{\left(w_{1}+w_{2}\right)}
$$

$i=1,2$, Outputs are called normalized firing strengths.

Layer 4: Every node $i$ in this layer is an adaptive node with a node function:

$$
04, i=w_{i} f_{i}=w_{i}\left(p_{i} x+q_{i} y+r_{i} z+S_{i}\right)
$$

wi is the normalized firing strength from layer 3.\{pi, qi, ri $\}$ is the parameter set of this node. These are referred to as consequent parameters.

Layer 5: The single node in this layer is a fixed node labeled sum, which computes the overall output as the summation of all incoming signals. Overall output $=05,1$ :

$$
\sum_{i} w_{i} f_{i}=\frac{\sum w_{i} f_{i}}{\sum w_{i}}
$$

The ANFIS can be trained by a hybrid learning algorithm in the forward pass the algorithm uses Least-squares method to identify the consequent parameters on layer 4, as shown in Table 5. In the backward pass the errors are propagated backward and the premise parameters are updated by gradient descent.

Table 5. ANFIS hybrid learning algorithm

\begin{tabular}{|l|l|l|}
\hline & Forward Pass & $\begin{array}{l}\text { Backward } \\
\text { Pass }\end{array}$ \\
\hline $\begin{array}{l}\text { Premise } \\
\text { Parameters }\end{array}$ & Fixed & $\begin{array}{l}\text { Gradient } \\
\text { Descent }\end{array}$ \\
\hline $\begin{array}{l}\text { Consequent } \\
\text { Parameters }\end{array}$ & $\begin{array}{l}\text { Least square } \\
\text { estimators }\end{array}$ & Fixed \\
\hline Signals & Node outputs & Error signal \\
\hline
\end{tabular}




\subsection{Fuzzy model for CNT infused copper electrode EDM}

The fuzzy logic is based on the identification of the fuzzy-set which represents the possible values of the variables. Fig. 3 shows the real inputs and real output. Fuzzy model described is for multiple input single output (MISO) system. In the present study, this MISO system includes three inputs and one output. These inputs are pulse on-time, pulse offtime and pulse current and the output is Surface Roughness of AISI D2 tool steel. Possible universe of discourse for the input parameters is given in Table 6.
Table 6. Identifying control factors and their levels

\begin{tabular}{|c|c|c|c|c|c|}
\hline Item & $\begin{array}{c}\text { Control } \\
\text { Factor }\end{array}$ & Units & $\begin{array}{c}\text { Level } \\
1\end{array}$ & $\begin{array}{c}\text { Level } \\
2\end{array}$ & $\begin{array}{c}\text { Level } \\
3\end{array}$ \\
\hline A & $\begin{array}{c}\text { Pulse- } \\
\text { on time }\end{array}$ & $\mu \mathrm{s}$ & 1 & 6 & 11 \\
\hline B & $\begin{array}{c}\text { Pulse- } \\
\text { off } \\
\text { time }\end{array}$ & $\mu \mathrm{s}$ & 1 & 4 & 7 \\
\hline $\mathrm{C}$ & $\begin{array}{c}\text { Pulse } \\
\text { current }\end{array}$ & Amp. & 3 & 4 & 5 \\
\hline
\end{tabular}

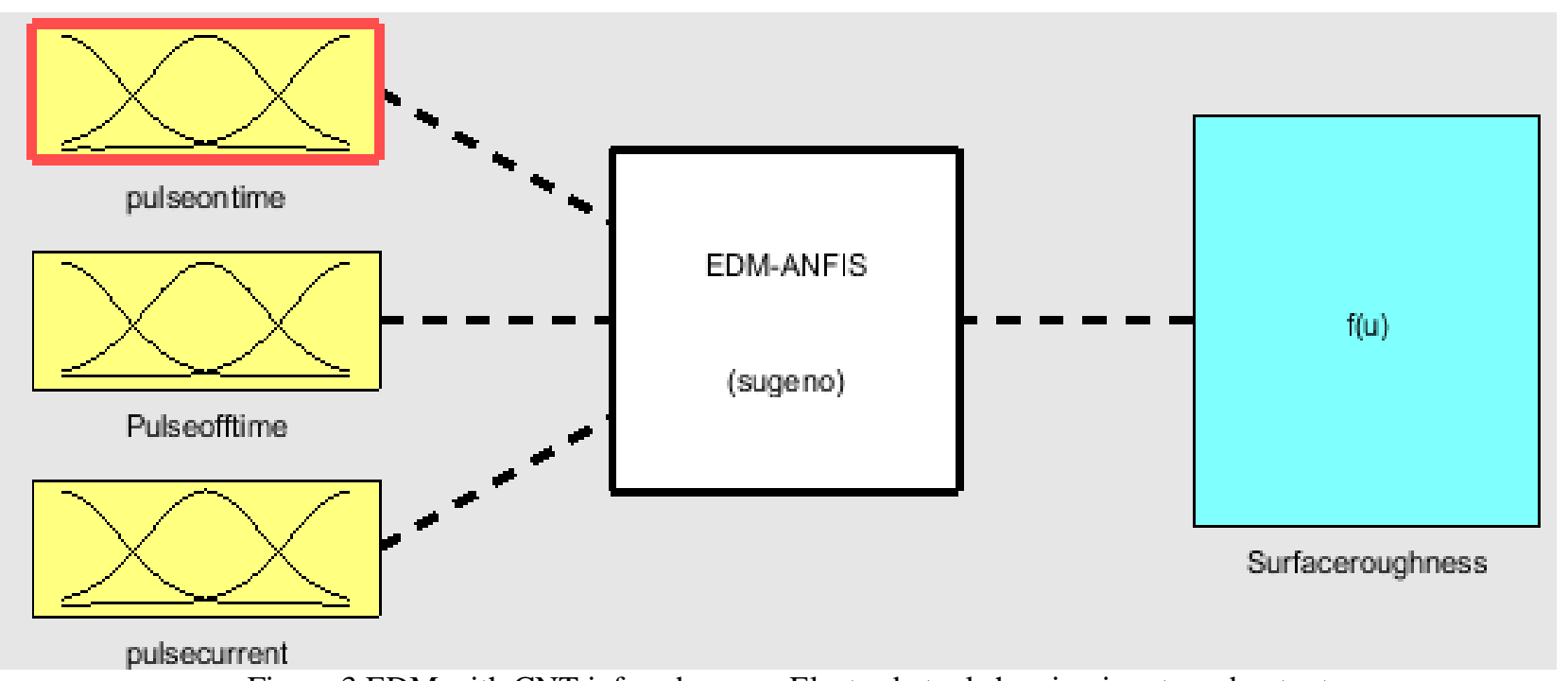

Figure.3 EDM with CNT infused copper Electrode tool showing inputs and output

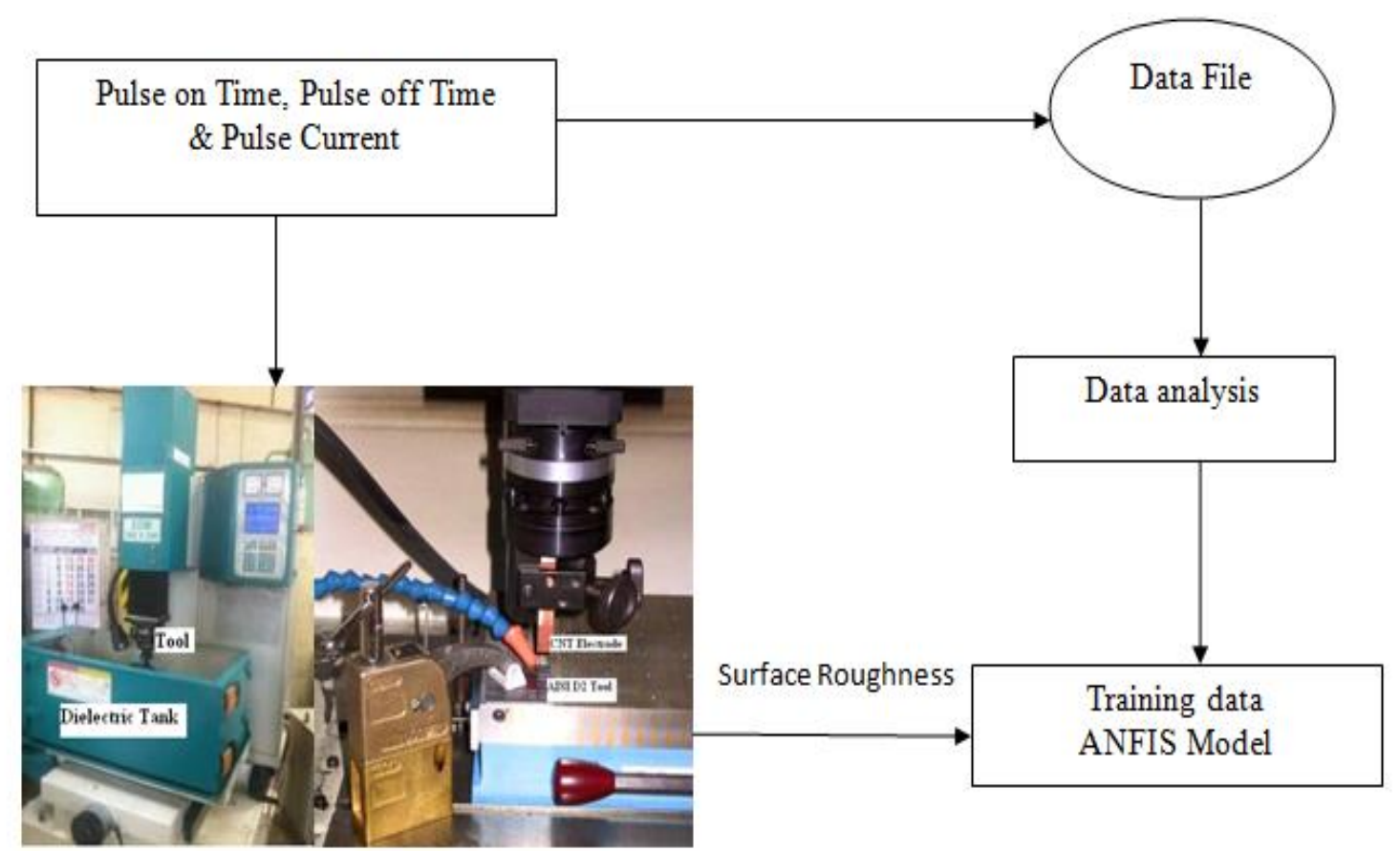

Figure.4 EDM process with CNT infused copper electrode setup for obtaining training data for ANFIS model 
The training data was established in ANFIS procedure by combination of inputs and the surface roughness output parameters were obtained with copper-CNT electrode based machining. The accuracy in calculation of the outputs of the neural network is purely based on the quality of training data. The EDM process with CNT infused copper electrode setup for obtaining training data for ANFIS model is shown in Fig.4.Using fuzzy logic tool box, training data adaption for the assessment of membership functions can be obtained. Fuzzy logic is a mathematical theory of inexact reasoning that allows modeling of the reasoning process of humans in linguistic terms (Hasmi [16]). It is very suitable for defining the relationship between system inputs and desired outputs. A fuzzy system is composed of a fuzzifier, an inference engine, a database, a rule base, and defuzzifier. In the study, the fuzzifier firstly uses membership functions to convert the crisp inputs into fuzzy sets, and then the inference engine performs a fuzzy reasoning on fuzzy rules to generate fuzzy values, then the defuzzifier converts these values into the crisp outputs.

The usage of fuzzy algorithms is not limited to fuzzy logic controllers. Fuzzy algorithms can be used equally well as nonlinear adaptation mechanisms, universal approximators or auxiliary units added to some conventional control solutions. It should also be noted that fuzzy controllers are very convenient as supervisory controllers. Sometimes, fuzzy logic algorithms are also used as modal or fuzzy state controllers. Despite the variety complexity of possible fuzzy controller structures, the basic form remains the same in all common types of controllers, consisting of:

- Input fuzzification (binary-to-fuzzy

$[\mathrm{B} / \mathrm{F}]$ conversion)

- Fuzzy rule base

- Inference engine

- Output defuzzification (fuzzy-to-binary [F/B] conversion)

The basic structure of a fuzzy controller is shown in Fig.5.

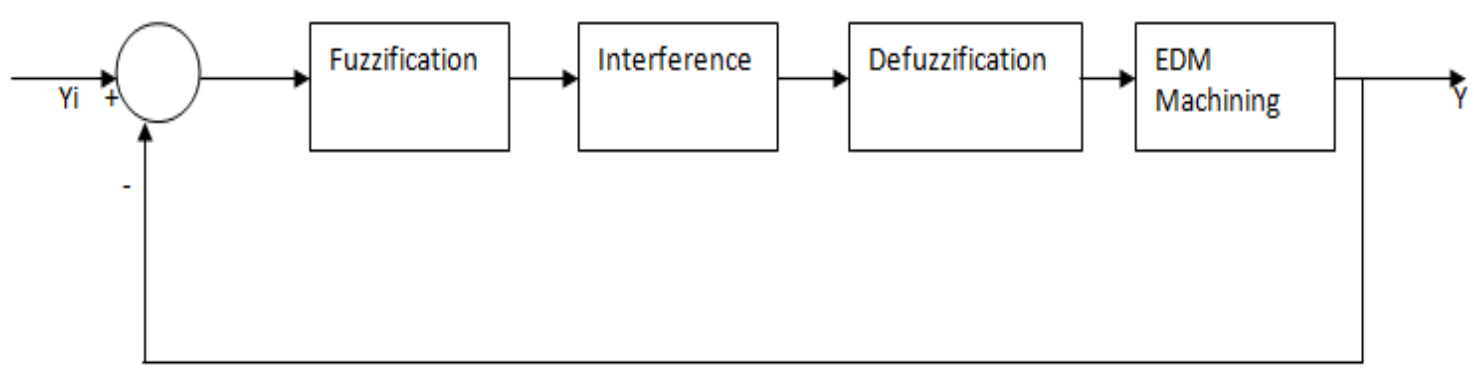

Figure.5 Block diagram of fuzzy control process with CNT copper electrode of EDM

Table 7. L9 orthogonal array of EDM parameters values

\begin{tabular}{|c|c|c|c|}
\hline Exp.No. & $\begin{array}{c}\text { Pulse-on } \\
\text { Time }(\mu \mathrm{s})\end{array}$ & $\begin{array}{c}\text { Pulse-off } \\
\text { Time }(\mu \mathrm{s})\end{array}$ & $\begin{array}{c}\text { Pulse Current } \\
(\text { Amp.) }\end{array}$ \\
\hline 1 & 1 & 1 & 3 \\
\hline 2 & 1 & 4 & 4 \\
\hline 3 & 1 & 7 & 5 \\
\hline 4 & 6 & 1 & 4 \\
\hline 5 & 6 & 4 & 5 \\
\hline 6 & 6 & 7 & 3 \\
\hline 7 & 11 & 1 & 5 \\
\hline 8 & 11 & 4 & 3 \\
\hline 9 & 11 & 7 & 4 \\
\hline
\end{tabular}

The next step involved is the machining on the EDM using the two electrodes. Before starting the machining, the surface of the electrode (where the sparking occurs) and workpiece are ground to a good finish, so as to obtain a flat surface for proper working conditions. The sparking area on the electrode is $6 \times 8 \mathrm{~mm}$, and the depth of machining is set to $1 \mathrm{~mm}$ for all, i.e. all machining done on the work surface will be of the same dimensions and volume. Before the machining is started, both the electrode and the workpiece are weighed using a precision weighing machine. After their weights are determined, the electrode and the workpiece are properly aligned on the worktable. . Post flawless alignment, the other required parameters are all set, which have been chosen and varied in accordance with L9 Taguchi array. This is depicted in Table 7. 
Once the machining is started, the time taken to machine the required depth in each case is duly noted. After the machining is over and once the dielectric is drained out properly, the electrode and the workpiece are removed, weighed again, and mounted back. The difference in the weight, before and after machining, is then calculated on both the workpiece material and the electrode for each set of machining and this gives the weight loss in $\mathrm{mg}$. After checking the weights, the workpiece and the tool are realigned and the next parameters are set in accordance with Table 8 . The loss in weight of tool and the copper electrode wear in the particular time interval gives the Electrode wear rate (EWR). Similarly, the loss in weight of the workpiece gives the Material removal rate (MRR). Material removal rate (MRR) and Electrode wear rate (EWR) can be calculated by the following formulae:

$$
\begin{aligned}
& M R R=\left(\frac{1000 W_{w}}{\rho_{w} T}\right) \\
& E W R=\left(\frac{1000 W_{e}}{\rho_{e} T}\right)
\end{aligned}
$$

where $W_{w}$ is the workpiece weight loss in grams, $\mathrm{W}_{\mathrm{e}}$ is the electrode weight loss in grams, $\rho_{w}$ is the workpiece material density in $\mathrm{gm} / \mathrm{cm}^{3}, \rho_{e}$ is the electrode material density in $\mathrm{gm} / \mathrm{cm}^{3}$ and $\mathrm{T}$ is the machining time in seconds. By using the above method EWR and MRR are calculated after every machining process, both for with and without CNT electrode cases. The surface roughness ( $\mathrm{Ra}$ ) of each machined workpiece is measured using the Talysurf (TR-200P).

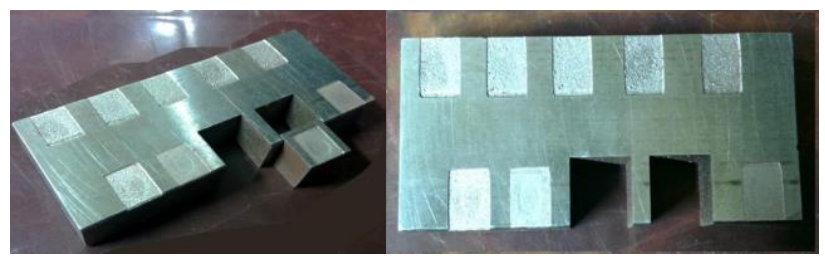

Figure.6 AISI D2 Tool steel workpiece after EDM machined with and without CNT electrode

\section{Experimental setup}

Experiments are conducted on die-sinking EDM machine, model type SEPL/TR/MO5 (refer to Fig.4). Experimental data based on the Taguchi design of experiments are collected to study the effect of various multi objective process parameters of CNT Infused copper electrode in EDM machine. The EDM machine has maximum 5 amp pulse current and $120 \mathrm{v}$ pulse voltage. These studies are undertaken to investigate the effects of pulse-on time, pulse-off time and pulse current on Surface roughness, MRR and EWR. AISI D2 Tool steel workpiece which is widely used in mold and die materials is machined using a copper induced CNT tool electrode and kerosene based dielectric fluids are used as the dielectric medium to strengthen the flow of electrons. The hardness obtained for the specimen is 55-62 HRC.

\subsection{Signal to noise ratio $(\mathrm{S} / \mathrm{N})$ analysis}

Signal to noise ratios $(\mathrm{S} / \mathrm{N})$ for each control factor are calculated, in order to minimize the variances in surface roughness values. The signals stipulate the effect on the average responses and noises calculated by the influence on the allowances from the average responses, which will disclose the sensitiveness of the experiment output of surface roughness to the noise factors. The appropriate $\mathrm{S} / \mathrm{N}$ ratio must be chosen based on prior knowledge, expertise and understanding of the EDM process for each application. In this study, the $\mathrm{S} / \mathrm{N}$ ratio is chosen based on the logical criterion the smallerthe-better, to minimize the responses and it is calculated according to Eq. (1):

$$
\frac{S}{N} \operatorname{Ratio}(\eta)=-10 \log 10 \frac{1}{n} \sum_{i=1}^{n} y^{2}
$$

\begin{tabular}{|c|c|c|c|}
\hline & \multicolumn{3}{|c|}{ Without CNT Electrode } \\
\hline $\begin{array}{l}\text { Exp. } \\
\text { No. }\end{array}$ & $\begin{array}{c}\text { Surface } \\
\text { Roughness (Ra) } \\
\mu \mathrm{m}\end{array}$ & $\begin{array}{c}\text { MRR } \\
\left(\mathrm{mm}^{3} / \mathrm{s}\right)\end{array}$ & $\begin{array}{c}\text { EWR } \\
\left(\mathrm{mm}^{3} / \mathrm{s}\right)\end{array}$ \\
\hline 1 & 4.307 & 0.083 & 0.0105 \\
\hline 2 & 4.079 & 0.051 & 0.0037 \\
\hline 3 & 3.664 & 0.015 & 0.0009 \\
\hline 4 & 2.738 & 0.016 & 0.0019 \\
\hline 5 & 3.377 & 0.035 & 0.0027 \\
\hline 6 & 4.876 & 0.031 & 0.0027 \\
\hline 7 & 1.472 & 0.006 & 0.0005 \\
\hline 8 & 1.577 & 0.004 & 0.0004 \\
\hline \multirow[t]{2}{*}{9} & 1.842 & 0.006 & 0.0004 \\
\hline & \multicolumn{3}{|c|}{ With CNT Electrode } \\
\hline $\begin{array}{l}\text { Exp. } \\
\text { No. }\end{array}$ & $\begin{array}{c}\text { Surface } \\
\text { Roughness (Ra) } \\
\mu \mathrm{m}\end{array}$ & $\begin{array}{c}\text { MRR } \\
\left(\mathrm{mm}^{3} / \mathrm{s}\right)\end{array}$ & $\begin{array}{c}\text { EWR } \\
\left(\mathrm{mm}^{3} / \mathrm{s}\right)\end{array}$ \\
\hline 1 & 4.025 & 0.118 & 0.009 \\
\hline 2 & 3.996 & 0.050 & 0.003 \\
\hline 3 & 3.338 & 0.017 & 0.001 \\
\hline 4 & 2.679 & 0.019 & 0.001 \\
\hline 5 & 2.943 & 0.042 & 0.002 \\
\hline 6 & 3.013 & 0.034 & 0.002 \\
\hline 7 & 1.221 & 0.016 & 0.000 \\
\hline 8 & 1.432 & 0.009 & 0.000 \\
\hline 9 & 1.558 & 0.008 & 0.000 \\
\hline
\end{tabular}

Table 8. Experimental results 

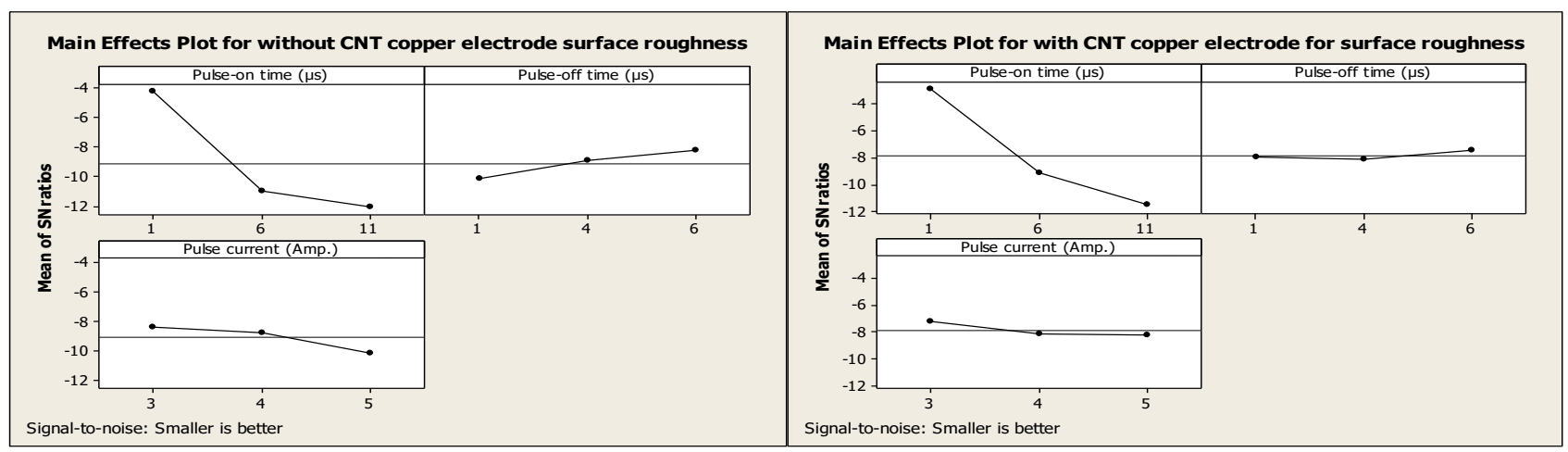

Figure.7 Factor effect diagram for without CNT copper electrode (left) with CNT (right) surface roughness

Where, $n$ is the number of repetitions that in this study is equal to 2 and $y$ is the surface roughness value for the $i$-th experiment. The values of MRR and EWR are calculated based on Eqs.5 and 6 for all the experimental work.

The Taguchi experimental results are summarized in Table 8 and factor effect diagram is presented in Fig.7. The lowest surface roughness is achieved when pulse-on time, pulse- off time and pulse current are chosen according to level of 3,1 and 3 , respectively for without and with CNT induced copper electrode.

The predicted $\mathrm{S} / \mathrm{N}$ ratio (í) using the optimal levels of the parameters can be calculated as:

$$
\dot{\eta}=\eta_{m}+\sum_{i=1}^{p} \eta_{i-} \eta_{m}
$$

In Eq.(8), $\eta_{m}$ - Total mean of $\mathrm{S} / \mathrm{N}$ ratio, $\eta_{i}$ - mean of $\mathrm{S} / \mathrm{N}$ ratio at the optimum level and $\mathrm{p}$ is the number of main parameters that significantly affect the performance.

For without CNT induced copper electrode, the predicted surface roughness ( $\mathrm{Ra}$ ) is $0.21 \mu \mathrm{m}$ and actual surface roughness $(\mathrm{Ra})$ is $1.472 \mu \mathrm{m}$. For with CNT copper electrode, the predicted surface roughness (Ra) is $0.24 \mu \mathrm{m}$ and actual surface roughness $(\mathrm{Ra})$ is $1.221 \mu \mathrm{m}$. In the surface finish analysis, the main variables are pulse-on time, pulse-off time and pulse current. Among these three parameters, in optimization technique, pulse on-time is ranked as first one for both the cases which influences the surface finish characteristics of AISI D2 tool steel hard materials.

\section{Fuzzy logic approach}

Fuzzy logic is a mathematical theory of inexact reasoning that allows modeling of the reasoning process of humans in linguistic terms (Yih-fong Tzeng [15]). It is suitable for defining the relationship between system inputs and desired outputs. A fuzzy system is composed of a fuzzifier, an inference engine, a database, a rule base, and defuzzifier. In the study, the fuzzifier firstly uses membership functions to convert the crisp inputs into fuzzy sets, and then the inference engine performs a fuzzy reasoning on fuzzy rules to generate fuzzy values, and then the defuzzifier converts these values into the crisp outputs.

For the prediction of output parameter surface roughness, the EDM process is modeled using three input parameters such as pulse on-time, pulse offtime and pulse current. The first step in establishing the algorithm for fuzzy model is to choose the shape of the fuzzy membership function or fuzzy sets of the process variables. The fuzzy expressions for different input parameters and output parameters are shown in Figs 8 and 9.

\subsection{Degree of membership functions}

The first stage in the application of fuzzy logic to model the EDM process is identifying the variation ranges of input and output variables. Then the range of each process variable is divided into groups of fuzzy subsets. Each fuzzy sub set is given a proper name and assigned a membership function. The membership function is assigned without depending on the results of the experiments. In general, membership functions are classified into trapezoidal, triangular, square, their combinations etc. In the present work triangular membership functions are selected for fuzzy input and output parameters. The pulse on-time of 1 to $11 \mu \mathrm{s}$ can be divided into three fuzzy sets as $\{$ low (mf1), medium (mf2), high (mf3) $\}$ and is $S=\left\{S_{1}, S_{2}, S_{3}\right\}$ expressed in vector form. The degree of membership function of pulse on-time is shown in Fig.8.

The membership function of pulse-on time in equation form is listed as follows:

$$
S_{1}(x)=\frac{6-x}{10}, x \in(1,6)
$$




$$
S_{2}(x)=\left\{\begin{array}{c}
(x-1) / 10 \\
(11-x) / 10,
\end{array} \quad x \in(1,6) \& x \in(6,11) \quad(10) \quad S_{3}(x)=\frac{x-11}{10}, x \in(6,11)\right.
$$

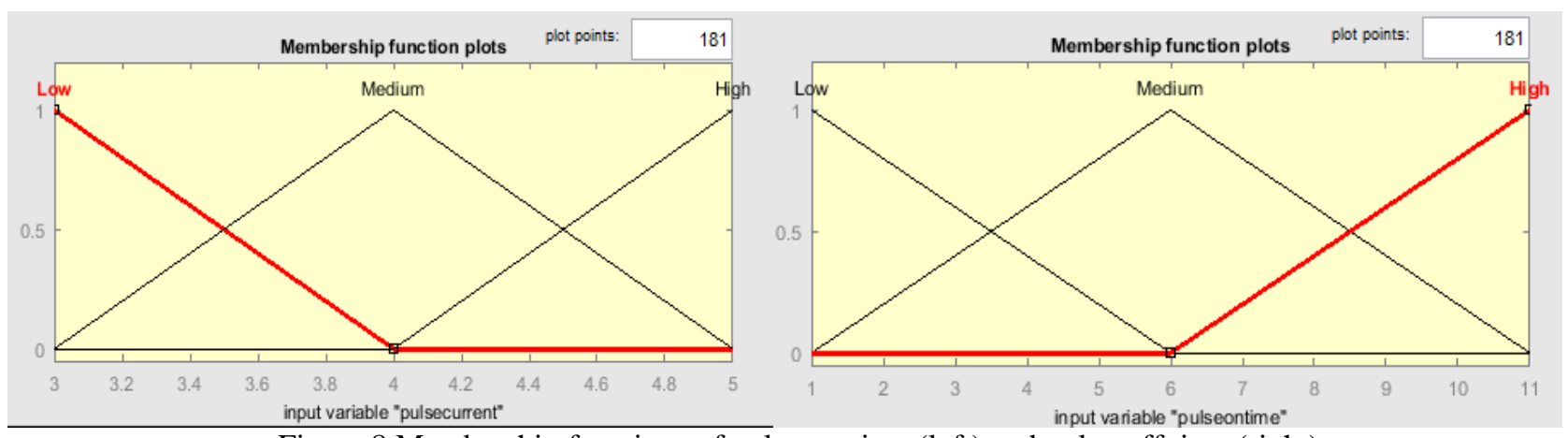

Figure. 8 Membership functions of pulse-on time (left) and pulse-off time (right)

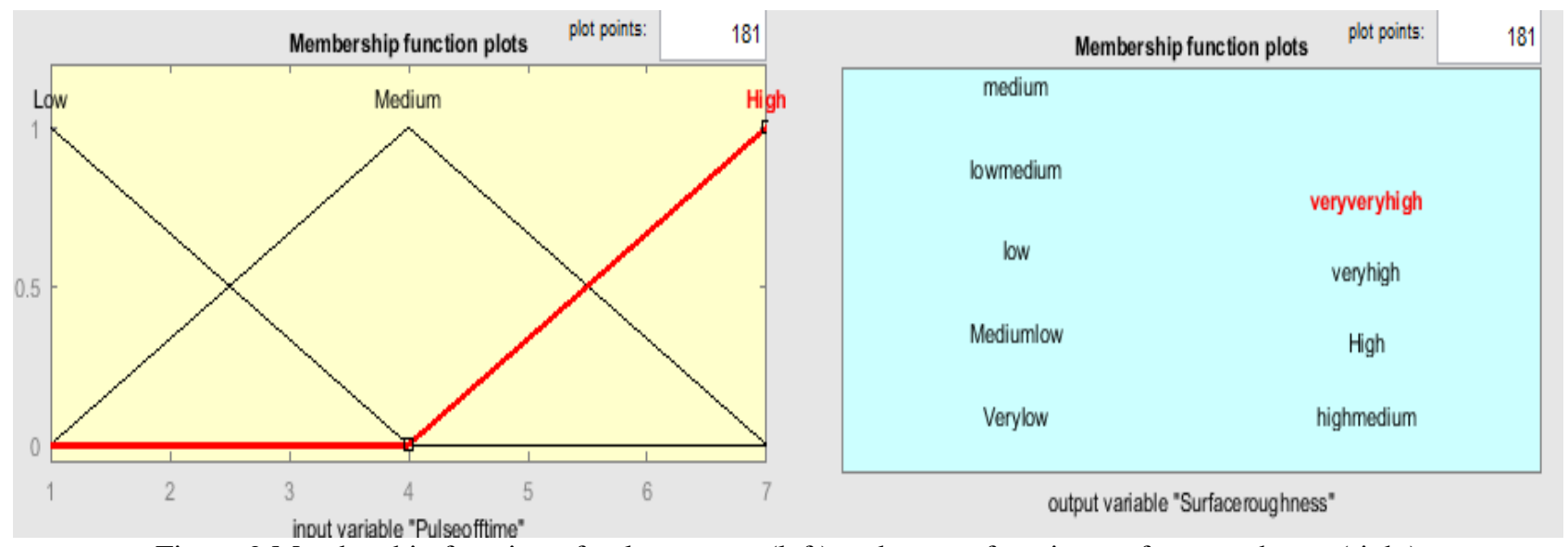

Figure.9 Membership function of pulse current (left) and output function surface roughness (right)

The range of pulse off-time is 1 to $5 \mu$ s and is divided into three fuzzy sets as \{low, medium, high and is $F=\{F 1, F 2, F 3\}$ expressed in vector form. The degree of membership function of pulse offtime is shown in Fig 8. Similarly the range of pulse current is 3 to $5 \mathrm{amp}$ and is divided into three fuzzy sets as $\{$ low, medium, high $\}$ and is $D=\{D 1, D 2$, D3\} expressed in vector form. The degree of membership function of pulse current is shown in Fig 9. The output parameter surface roughness is 1.22 to $4.025 \mu \mathrm{m}$ and is divided into nine fuzzy sets, as $\mathrm{Ra}=\{\mathrm{Ra} 1, \mathrm{Ra} 2, \mathrm{Ra} 3, \ldots \mathrm{Ra} 9\}$ expressed in vector form. The degree of membership function of surface roughness is shown in Fig 9.

\subsection{Fuzzy rule}

Fuzzy values are determined by the membership functions that define the degree of membership of an object in a fuzzy set [15]. However, so far there has been no standard method of choosing the proper shape of the membership functions for the fuzzy sets of the control variables. Trial and error methods are usually exercised. Based on the fuzzy rules, the Sugeno implication method is employed for the fuzzy inference reasoning in this study. The relationship between input and the output in fuzzy system is characterized by a set of linguistic statements which are called fuzzy rules. They are defined based on the experimental work, gained expertise and engineering knowledge. One experiment results in one fuzzy rule. If all the fuzzy rules are saved in a database, a fuzzy rule base will be established. The number of fuzzy rules in fuzzy system is related to the number of fuzzy sets for each input variable. In this study 9 fuzzy rules are established. The fuzzy rules can be expressed by:

$$
\begin{aligned}
& \mathrm{F}\left(\mathrm{S}_{\mathrm{i}}, \mathrm{F}_{\mathrm{i}}, \mathrm{D}_{\mathrm{i}}\right)-\mathrm{Ra} \\
& \quad(i, j, k \varepsilon[1,3] ; p, q, r \varepsilon[1,5] ; I, j, k, p, q, r, \varepsilon, z)
\end{aligned}
$$

Few examples of the fuzzy rules in linguistic form are shown in Fig.10 given below.

Rule 1: If pulse on-time is low, pulse off-time is low and pulse current is low, then Surface roughness is very low.

Rule 2: If pulse on-time is medium, pulse offtime is medium and pulse current is medium, then Surface roughness is medium. 
Rule 3: If pulse on-time is high, pulse off-time is high and pulse current is high, then Surface roughness is high etc.

These rules are formed based on the experimental results presented in Table 8 . The result of fuzzy inference is a fuzzy output set. On the other hand, every control task will imply the existence of crisp value at the fuzzy controller output. The procedure which extracts crisp output value from a fuzzy output set is called defuzzification. There are various types of defuzzification. The singleton method of defuzzification is adopted to predict the output parameters. This was carried out with the help of fuzzy logic controller in the MATLAB Tool Box.

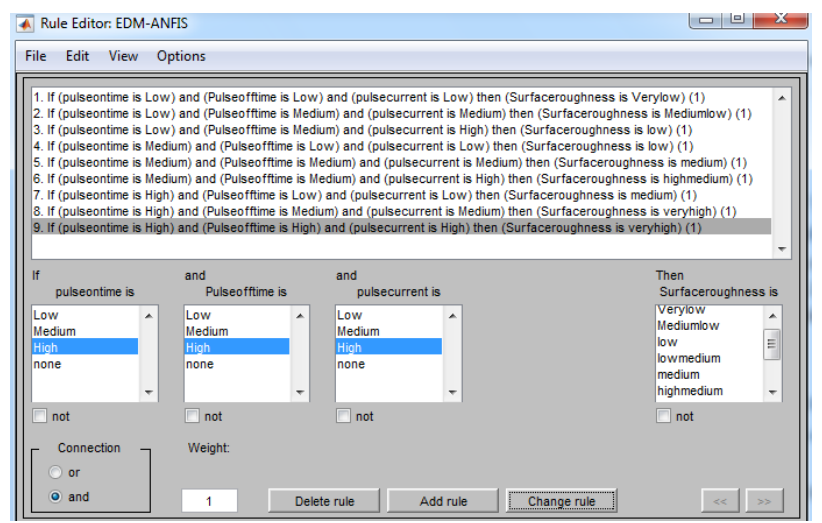

Figure.10 ANFIS Sugeno type rule editor

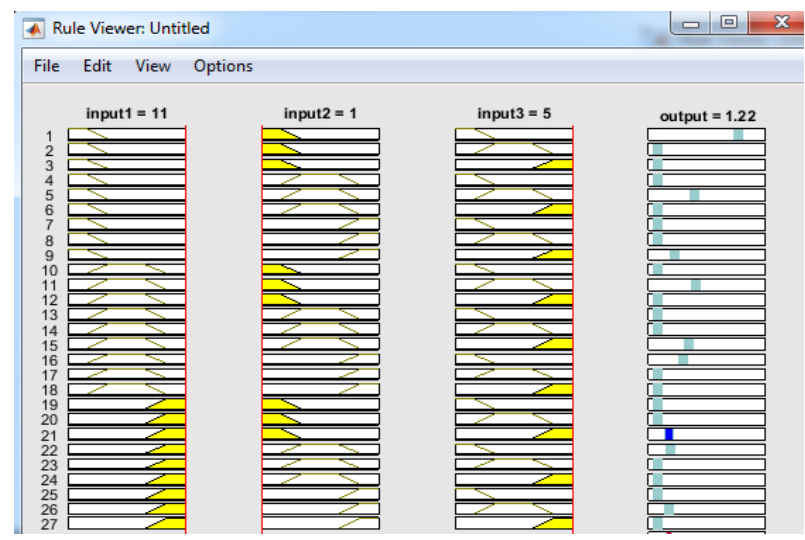

Figure.11 Fuzzy logic rule viewer results for machining with CNT

Graphical presentation of the fuzzy logic reasoning procedure for EDM machining results of Taguchi design of experiments of 9 experiments using Matlab software is shown in Fig.10 in which rows represent the 9 rules and columns are the threeinputs/one-output variables. The locations of triangles indicate the determined fuzzy sets for each input value and single line represents output values. The height of the darkened area in each triangle corresponds to the fuzzy membership value for that fuzzy set. For CNT based machining, the input normalized value of dimensional precision which belongs to the fuzzy sets $\mathrm{N}, \mathrm{F}$ and $\mathrm{d}$ corresponds to fuzzy rules $1-9$. The defuzzified output that gives the final surface roughness value is calculated as $1.22 \mu \mathrm{m}$ from the combined darkened areas shown in the bottom of Ra column. It is noted that the larger the dimensional precision and accuracy are, the larger the resulting surface roughness would be, that means that the parameter designed with higher machining parameters leads to better dimensional precision and accuracy of machined products.

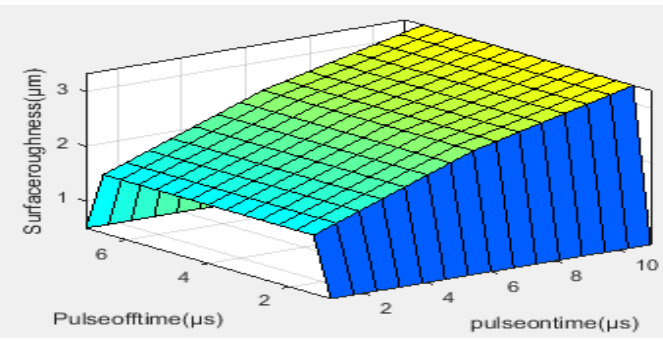

(a)

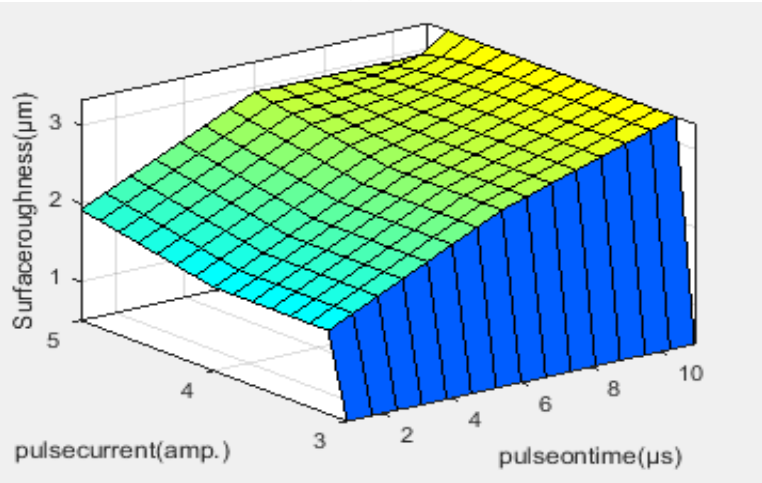

(b)

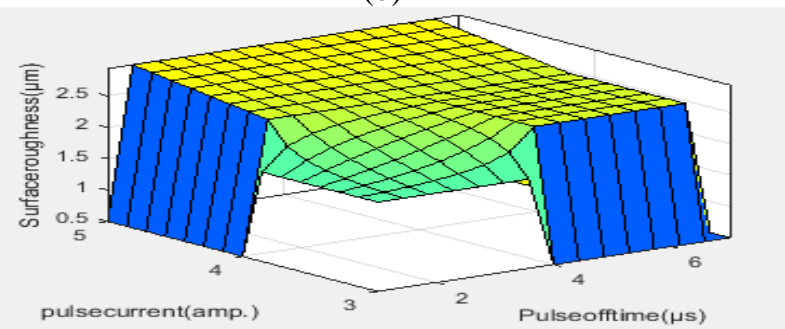

(c)

Figure.12 3D Surface fuzzy model shows surface roughness on (a) pulse on-time and pulse off-time, (b) Pulse on-time and Pulse current, and (c) pulse current and pulse off-time

Figure 12 shows combinations of machining variables with surface roughness. Different combination input values are used to obtain the minimum surface roughness. The printed screen of Sugeno type fuzzy tool box of Matlab which shows the minimum surface roughness and that pulse current is low and pulse on time is high. Twenty validation runs were estimated using ANFIS model 


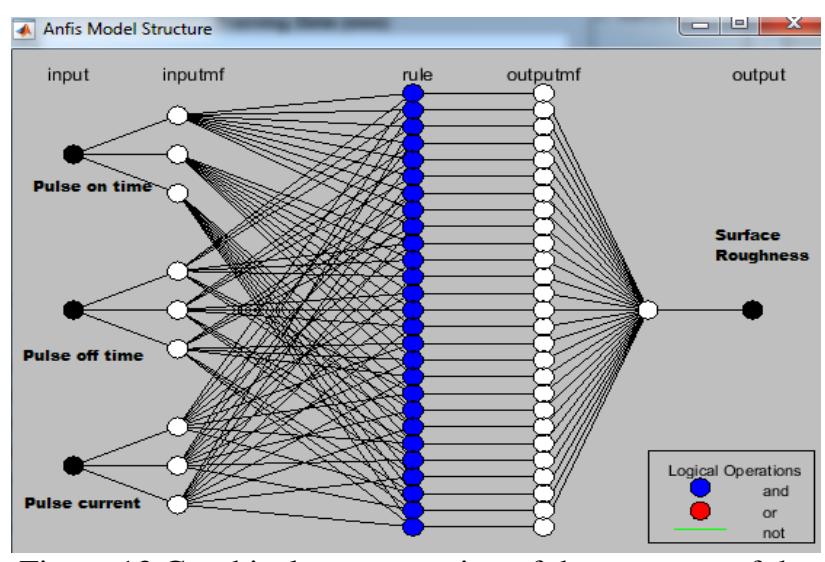

Figure.13 Graphical representation of the structure of the ANFIS controller using MATLAB

result. The output values came close to predicted values with an error of less than 5\%.

To use ANFIS for classification problem, the designer needs to perform the following steps:

1. Design a Sugeno FIS appropriate for the classification problem.

2. Optimize by hand the FIS, given actual input classification data.

3. Set up training and testing matrices. The training and testing matrices will be composed of inputs and the desired classification corresponding to those inputs.

4. Run the ANFIS algorithm on the training data.

5. Test the results using the testing data.

The ANFIS Matlab model structure shown in Figure 13 has three inputs: pulse on-time (x), pulse off-time (y) and pulse current (z) with each one having three triangular membership functions of low, medium and high values. The 9 fuzzy rules are formulated with 9 output singletron membership function using Sugeno type Fuzzy interference system.

The surface roughness predictions of the developed ANFIS model are presented as a function of the experimentally determined values. It is to be noted that the comparison was made using values only from the test data set, which was not introduced to the ANFIS during the training process. In order to assess the accuracy of ANFIS predictions, graphic is provided with a straight line indicating perfect prediction. As shown in Fig.14, the ANFIS predictions for the surface roughness came close with an error of less than $5 \%$.
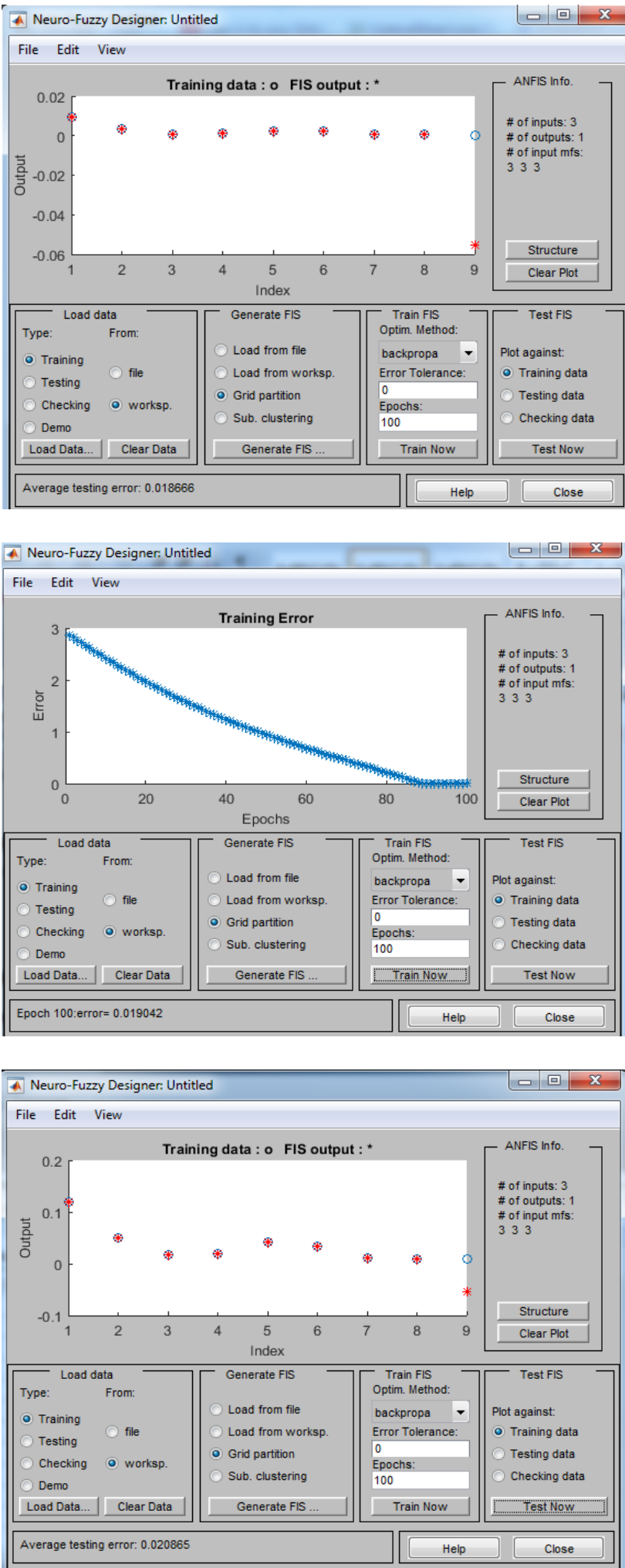

Figure.14 ANFIS predictions during the training process

\subsection{ANOVA analysis}

Analysis of variance (ANOVA) test was performed to identify the most effective process parameters which affect the surface roughness of AISI D2 tool steel materials. The ANOVA table for 
with and without $\mathrm{CNT}$ alternatives and the $\mathrm{S} / \mathrm{N}$ ratio are listed in Tables 9 and 10, respectively. Precision of parameter calculation is purely based on the number of independent samples of information which can be determined by degree of freedom (DOF). The degree of freedom is equal to the number of experiments minus the number of additional parameters estimated for calculation. The results of ANOVA indicate that pulse-on time process parameters have significant contribution onto the achieved surface roughness values. In addition, a numerical model by a design expert has been developed and the analysis of variance (ANOVA) by the design expert is presented and compared. The R2 coefficient indicates the good fit of the model goodness of fit for the model. In this case, the value of the coefficient $(\mathrm{R} 2=0.9773$ ) indicates that $97.73 \%$ of the total variability is explained by the model after considering all the significant factors. The F-value of 42.04 implies that the model is significant.

Table 9. The ANOVA for the surface roughness without CNT copper electrode

\begin{tabular}{|l|c|c|c|c|c|c|}
\hline \multicolumn{1}{|c|}{ Machining parameters } & $\begin{array}{c}\text { Degree of } \\
\text { Freedom (f) }\end{array}$ & $\begin{array}{c}\text { Sum of Squares } \\
\left(\mathrm{SS}_{\mathrm{A}}\right)\end{array}$ & $\begin{array}{c}\text { Variance } \\
\left(\mathrm{V}_{\mathrm{A}}\right)\end{array}$ & $\mathrm{FA}_{\mathrm{o}}$ & $\mathrm{P}$ & Contribution (\%) \\
\hline Pulse-on time $(\mu \mathrm{s})$ & 2 & 9.953 & 4.976 & 9.7 & 0.09 & 78.74 \\
\hline Pulse-off time $(\mu \mathrm{s})$ & 2 & 0.618 & 0.309 & 0.60 & 0.62 & 4.85 \\
\hline Pulse current (Amp.) & 2 & 1.053 & 0.526 & 1.03 & 0.49 & 8.33 \\
\hline Error & 2 & 1.022 & 0.511 & & & 8.08 \\
\hline Total & 8 & 12.642 & & & & 100 \\
\hline
\end{tabular}

*Significant $\mathrm{S}=0.714832 \quad \mathrm{R}-\mathrm{Sq}=91.92 \% \quad \mathrm{R}-\mathrm{Sq}(\operatorname{adj})=67.68 \%$

Table 10. The ANOVA for the surface roughness with CNT induced copper electrode

\begin{tabular}{|l|c|c|c|c|c|c|}
\hline \multicolumn{1}{|c|}{ Machining parameters } & $\begin{array}{c}\text { Degree of } \\
\text { Freedom }(\mathrm{f})\end{array}$ & $\begin{array}{c}\text { Sum of Squares } \\
\left(\mathrm{SS}_{\mathrm{A}}\right)\end{array}$ & $\begin{array}{c}\text { Variance } \\
\left(\mathrm{V}_{\mathrm{A}}\right)\end{array}$ & $\mathrm{FA}_{\mathrm{o}}$ & $\mathrm{P}$ & Contribution (\%) \\
\hline Pulse-on time $(\mu \mathrm{s})$ & 2 & 8.6762 & 4.338 & 42.04 & 0.02 & 95.36 \\
\hline Pulse-off time $(\mu \mathrm{s})$ & 2 & 0.0458 & 0.022 & 0.22 & 0.81 & 0.59 \\
\hline Pulse current $(\mathrm{Amp})$. & 2 & 0.1697 & 0.084 & 0.82 & 0.54 & 1.96 \\
\hline Error & 2 & 0.2064 & 0.103 & & & 2.09 \\
\hline Total & 8 & 9.0982 & & & & 100 \\
\hline
\end{tabular}

*Significant $\mathrm{S}=0.321239 \mathrm{R}-\mathrm{Sq}=97.73 \% \quad \mathrm{R}-\mathrm{Sq}(\operatorname{adj})=90.93 \%$

Table 11. Results of confirmation tests for surface roughness and ANFIS for with CNT infused Electrode

\begin{tabular}{|c|c|c|c|}
\hline \multirow{2}{*}{} & \multirow{2}{*}{ Initial Design } & \multicolumn{2}{|c|}{ Optimal Design } \\
\cline { 3 - 4 } & & ANFIS Prediction & Taguchi Experiment \\
\hline Setting level & A1B1C1 & A3B1C3 & A3B1C3 \\
\hline $\begin{array}{c}\text { Surface } \\
\text { Roughness }(\mu \mathrm{m})\end{array}$ & 4.025 & 1.220 & 1.221 \\
\hline MRR $\left(\mathrm{mm}^{3} / \mathrm{s}\right)$ & 0.119 & 0.016 & 0.0162 \\
\hline EWR $\left(\mathrm{mm}^{3} / \mathrm{s}\right)$ & 0.00954 & 0.00043 & 0.00047 \\
\hline
\end{tabular}

\subsection{Confirmation test}

The confirmation experiment is the final step in the first iteration of the design of experimental process. The purpose of the confirmation experiment is to validate the conclusions drawn during the ANFIS. It is conducted by setting the process parameters at the optimum level of A1B1C1. Pulse-on time $1 \mu \mathrm{s}$, pulse-off time $6 \mu \mathrm{s}$ and pulse current $3 \mathrm{amp}$ are set as optimum parameters and the actual surface roughness obtained with CNT copper electrode is $4.025 \mu \mathrm{m}$ to initial design $3.338 \mu \mathrm{m}$. 
Improvement in surface roughness shows that the ANFIS is used to predict the output parameters and they are compared closely with experimental values which increases the accuracy of results further.

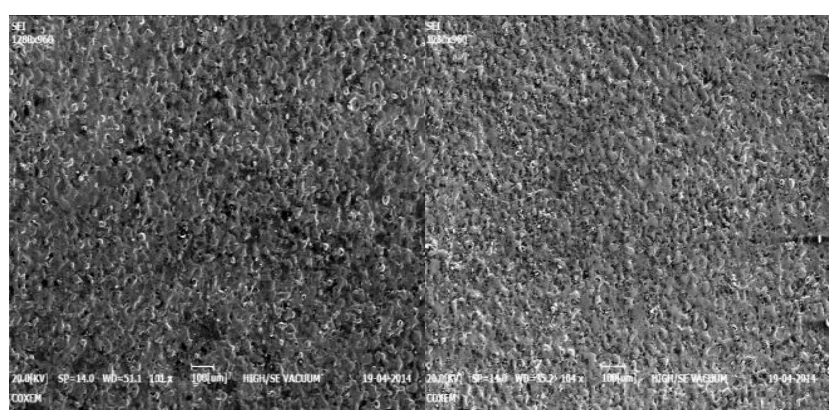

Figure.15 SEM analysis of EDM Machined workpiece using Pure Copper Electrode (left) and CNT Copper Electrode (right) for sample 1

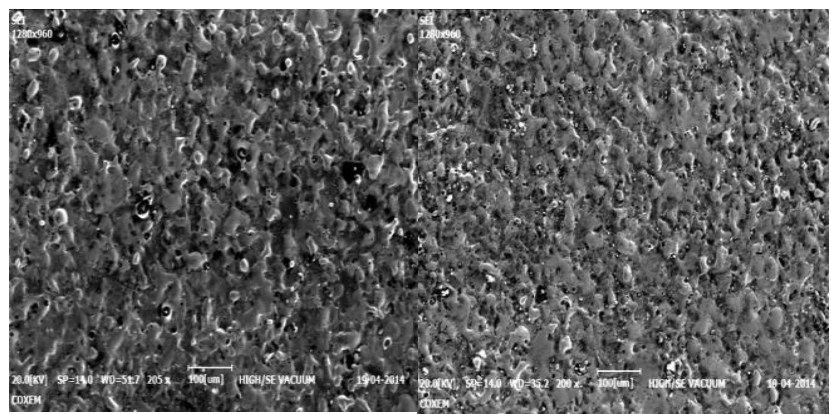

Figure.16 SEM analysis of EDM Machined workpiece using Pure Copper Electrode (left) and CNT Copper Electrode (right) for sample 2

\subsection{Surface Morphology of AISI D2 Tool steel after CNT based machining}

The surface texture is composed of a random array of overlapping craters or cups after machining. During each electrical discharge, intense heat is generated and a crater is formed on the workpiece. Some of the molten material produced by the discharge is carried away by the dielectric fluid circulation. The remaining melt resolidifies to form an undulating terrain. In order to assess the resulting surface finish, a SEM analysis of the surface topography of the EDM machined surface is conducted at different magnifications. From the comparison of two cases based on Fig.15 and Fig.16, it is evident that the machined area under CNT electrode is more, even with particles of fairly uniform size spread evenly, and has a better surface finish. The number of white spots from the white layer formation which represents the unwanted particles resulting out of the heat interaction is much lesser as compared to the machined area without CNT. From the comparison based on SEM image the hot spots resulting out of sparking and burning of metal are lesser in the case of the surface machined by CNT infused electrode as compared to the normal $\mathrm{Cu}$ electrodes. The microcracks are also visibly lesser in number making the former surface a better choice.

\section{Conclusions}

The ANFIS modelling of surface roughness with CNT based machining of AISI D2 tool steel material was done with nano and plain electrodes. The Taguchi design of experimental techniques was adopted to conduct the experimental work. It is en found that results generated by the designed model are close to the experimental results with $96.20 \%$ accuracy. The present study shows that the ANFIS is a technique that can be used efficiently to optimize the machining of tool steel with CNT. It is believed that this approach can be applied to optimize many other similar manufacturing processes. With this level of accuracy, the model can be used by any practicing engineer who would like to get quick answers for on-line intelligent control and optimization. This system is found to be very flexible and easy to comprehend and hence can be considered as an alternative to conventional modelling techniques. The analytical results are summarized as follows:

1. The results of ANOVA indicate that pulse-on time process parameters have significant contribution to the surface roughness values. In addition, a numerical model by a design expert has been developed and the analysis of variance (ANOVA) by the design expert is presented. The R2 coefficient indicates the good fit for the model. In this case, the value of the coefficient $(\mathrm{R} 2=0.9773)$ indicates that $97.73 \%$ of the total variability is explained by the model after considering the significant factors. The F-value of 42.04 implies that the model is significant.

2. The regression empirical model was developed based on the relationship between surface roughness with pulse current, pulse-on duration and pulse voltage of EDM process. The R2 value of regression model for with CNT is 0.706 and for without CNT is 0.652 . Similarly, regression model for with CNT based MRR is 0.535 and for without CNT is 0.423 . The high R2 indicated that the better model fit all. The data very well using CNT based EDM machining.

3. The developed fuzzy logic model used to evaluate the surface roughness produced by EDM machining with CNT, has $2.009 \mu \mathrm{m}$ with low prediction errors of $1.07 \%$ to $28.7 \%$. The fuzzy 
model based approach improves the predicted model for better process parameters accurately and precisely.

4. The adaptive neuro-fuzzy inference system method based on L9 orthogonal array experimentation is used for predicting surface roughness, for both with and without CNT based EDM process. It is believed that this approach can be applied to optimize many other manufacturing processes. With this level of accuracy, the model can be used by the practicing engineer who would like to get quick answers for on-line intelligent control and optimization.

5.Surface topology analyzed through the SEM analysis depicts a distinctive improvement in the surface characteristics and reduction in the white spots, hotspots and the micro-cracks. It is clear that the specimens sparked using CNT-infused electrodes have better machining characteristics and surface finish as compared with specimens sparked without CNT.

For case without CNT induced copper electrode, the predicted surface roughness (Ra) is $0.21 \mu \mathrm{m}$ and actual surface roughness (Ra) is $1.472 \mu \mathrm{m}$. For cases with CNT copper electrode, the predicted surface roughness (Ra) is $0.24 \mu \mathrm{m}$ and actual surface roughness $(\mathrm{Ra})$ is $1.221 \mu \mathrm{m}$. In the surface finish analysis, the main variables are pulse-on time, pulse-off time and pulse current and among these three parameters in optimization technique, pulse-on time is ranked first in both the cases which influences the surface finish characteristics of AISI D2 tool steel hard materials.

\section{Future Scope}

The next step following this proof-of-principle study is to export the techniques to other electrode materials to investigate the machining characteristics of materials on the EDM process and check the improvement in machinability. Further, by varying the wt. percentage of CNT it is possible to compare the machining characteristics of different hard work piece materials.

\section{References}

[1] M. Kiyak and A. C. Aakır, "Examination of machining parameters on surface roughness in EDM of tool steel", J. of Mater. Proc. Tech., Vol. 191, pp.141-144, 2007.

[2] C. H. Che Haron, J. A. Ghani, Y. Burhanuddin, Y. K. Seong, and C. Y. Swee, "Copper and graphite electrodes performance in electricaldischarge machining of XW42 tool steel", J. of Mater. Proc. Tech., Vol. 201, pp. 570-573, 2008.
[3] Y.H. Guu, "AFM surface imaging of AISI D2 tool steel machined by the EDM process", Applied Surface Science, Vol. 242, pp. 245-250, 2005.

[4] Y. H. Guu, H. Hocheng, C. Y. Chou, and C. S. Deng, "Effect of electrical discharge machining on surface characteristics and machining damage of AISI D2 tool steel", Materials Science and Engineering, A358, pp.37-43, 2003.

[5] S. Prabhu and B. K. Vinayagam, "Analysis of surface characteristics of AISI D2 tool steel material using electric discharge machining process with single-wall carbon nanotubes", Int. J. of Mach. and Machina. of Mater., Vol. 11, No.1/2 pp. 99-119, 2011.

[6] K. C. Lee, S. J. Ho, and S. Y. Ho, "Accurate estimation of surface roughness from texture features of the surface image using an adaptive neuro-fuzzy inference system", Prec. Eng., Vol.29, pp.95-100, 2005.

[7] K. Maji and D. K. Pratihar, "Forward and reverse mappings of electrical discharge machining process using adaptive networkbased fuzzy inference system", Expert Sys. with Appl, Vol.37, pp.8566-8574, 2010.

[8] K. M. Tsai and P. J. Wang, "Prediction on surface finish in electrical discharge machining based upon neural network models", Int. J of Mach Tools and Manuf., Vol.41, pp.1385-1403, 2001.

[9] O. Yilmaz, O. Eyercioglu, N. N. Z. Gindy, "A user friendly fuzzy based system for the selection of electro discharge machining process parameters", $J$. of Mat. Proc. Tech., Vol.72, pp. 363-371, 2006.

[10] U. Caydas, A. Hascalik, and S. Ekici, "An adaptive neuro-fuzzy inference system (ANFIS) model for wire-EDM", Expert Sys. with Appl., Vol.36, pp.6135-6139, 2009.

[11] K. M. Tsai and P. J. Wang, "Comparison of neural network models on material removal rate in electric discharge machining", J. of Mat. Proc. Tech., Vol.117. pp.111-124, 2001.

[12] M. Dong and N. Wang, "Adaptive networkbased fuzzy inference system with leave-one-out cross-validation approach for prediction of surface roughness", Appl. Math Model, Vol.35, pp.1024-1035, 2011.

[13] D. Joshi, A. Mishra, and S. Anand, "ANFIS based knee angle prediction: An approach to design speed adaptive contralateral controlled AK prosthesis", Appl. Soft Comp., Vol.11, pp.4757-4765, 2011.

[14] S. N. Joshi and S. S. Pande, "Intelligent process modeling and optimization of die-sinking 
electric discharge machining", Appl. Soft Comp., Vol.11, pp.2743-2755, 2011.

[15] Y. Tzeng and F. Chen, "Multi-objective optimisation of high-speed electrical discharge machining process using a Taguchi fuzzy-based approach", Materials and Design, Vol.28, pp.1159-1168, 2007.

[16] K. Hashmi, M. A. El Baradie, and M. Ryan, "Fuzzy-logic based intelligent selection of machining based upon neural network models", Int. J. of Machi Tools \& Manuf., Vol. 41, pp.1385-1403, 1999.

[17] G. Skrabalak, M. Zybura-Skrabalak, and A. Ruszaj, "Building of rules base for fuzzy-logic control of the ECDM process", J. of Mat. Proc. Tech., Vol.149, pp.530-535, 2004.

[18] T. Rajasekaran, K. Palanikumar, and B. K. Vinayagam, "Application of fuzzy logic for modeling surface roughness in turning CFRP composites using CBN tool", Prod.. Eng. Res. Devel., Vol.5, pp.191-199, 2011.

[19] K. Hashmi, I. D. Graham, and B. Mills, "Fuzzy logic based data selection for the drilling process", J. of Mat. Proc. Tech., Vol.108, pp.5561,2000 . 